\title{
Relationship between amino acid sequence diversity and antimicrobial activity of Japanese quail cathelicidins
}

\section{Taichiro Ishige}

Tokyo Nogyo Daigaku https://orcid.org/0000-0001-8424-7833

Hiromi HARA

Tokyo Nogyo Daigaku

Takashi HIRANO

Tokyo Nogyo Daigaku

Tomohiro KONO

Tokyo Nogyo Daigaku

Kei HANZAWA ( $\nabla$ khanzawa@nodai.ac.jp )

https://orcid.org/0000-0002-6751-3447

Research article

Keywords: Amino acid substitution, antimicrobial activity, cathelicidin, host defense peptide, polymorphism, single nucleotide polymorphism

Posted Date: September 12th, 2019

DOI: https://doi.org/10.21203/rs.2.14418/v1

License: (c) (1) This work is licensed under a Creative Commons Attribution 4.0 International License. Read Full License 


\section{Abstract}

Background Japanese quail is a low-fat, meat-bird species exhibiting high disease resistance. Cathelicidins (CATHs) are host defense peptides conserved across numerous vertebrate species that play an important role in innate immunity. The activity of host defense peptides can be affected by amino acid substitutions. However, no polymorphisms in avian CATH genes have been reported to date. The aim of this study was to clarify the effect on antimicrobial activity of polymorphisms in CATHs. Results DNA for genomic analyses was extracted from the peripheral blood of 99 randomly selected quail from 6 inbred lines. A total of six, four, four, and six CjCATH3 , CjCATH2 , CjCATHB1, and CjCATH1 alleles were identified, respectively. Nine haplotypes, including four that were strain specific, were identified in alleles from the CjCATH3 , CjCATH2 , CjCATHB1 , and CjCATH1 loci. In addition, two and one amino acid substitutions (I145F, Q148H, and P245H) predicted by PROVEAN and PolyPhen-2 to have deleterious effects were detected at CjCATH2 and B1, respectively. Synthetic CjCATH2 and B1 peptides exhibited greater antibacterial activity against Escherichia coli than chicken CATH2 and B1, respectively. Furthermore, CjCATHB1*04 peptide exhibited less-potent antimicrobial activity than other CjCATHB1 peptides examined. Conclusions This is the first report of amino acid substitutions accompanied by changes in antibacterial activity in avian CATHs. These findings could be employed as indicators of improvements in the innate immune response in poultry.

\section{Background}

Host defense peptides (HDPs) are small cationic peptides conserved among all vertebrate species [1] that function as important mediators of innate immune responses [2]. Defensins and cathelicidins (CATHs) represent two major families of HDPs. CATHs are short ( $<40$ amino acid residues), cationic, amphipathic peptides that have been identified in a variety of vertebrate species [3]. CATHs are composed of a cathelin-like domain in the N-terminal region of the precursor. Although the CATH signal peptide and cathelin-like domain are highly conserved across species, the sequence of the $\mathrm{C}$-terminal mature peptide varies significantly [4].

CATHs play a number of important immunity-related roles, such as mediating heterophil chemotaxis, cytokine and chemokine production in monocytes, the inhibition of apoptosis, and binding and inactivation of lipopolysaccharide (LPS) [5]. CATHs exhibit antimicrobial activity against both grampositive and -negative bacteria, fungi, protozoa, and viruses [6,7]. CATHs also reportedly direct chemotaxis activity, induce chemokine release, modulate the differentiation of macrophages and dendritic cells, enhance phagocytosis, promote wound healing, and mobilize leukocytes via modulation of TLR activation [8].

CATH genes have been identified in 15 avian species [4,9,10]. The four CATH genes (GgCATH3, GgCATH2, GgCATHB1 and GgCATH1) of chickens are tightly clustered in a 7.5-kb region at the proximal end of chromosome 2 [11]. Similar to mammalian CATH genes, all GgCATH genes consist of four exons [4], the first three of which encode the 5 -untranslated region, signal peptide, and cathelin-like domain, whereas 
the fourth exon encodes both the mature peptide and 3'-untranslated region [4]. Transcription of GgCATH1, GgCATH2, and GgCATH3 mRNAs occurs primarily in the bone marrow, although transcripts of all three $\mathrm{GgCATH}$ s have also been detected in tissues of the digestive, respiratory, and urogenital tracts [12,13,14,15]. GgCATHB1 expression has been reported in epithelial cells [4], the bursa of Fabricius [15], as well as the jejunum, colon, thymus, and peripheral blood leukocytes $[14,16]$. Avian CATHs exhibit antimicrobial activity against both gram-positive bacteria such as Listeria monocytogenes and Staphylococcus aureus and gram-negative bacteria such as Escherichia coli and Salmonella typhimurium $[17,18]$. Various studies have shown that GgCATH1, GgCATH2, and GgCATH3 play a range of immunoregulatory roles, including suppression of LPS-induced production of proinflammatory cytokines via binding to LPS $[11,18,19]$ and induction of specific chemokines [19].

In the genome of Japanese quail, we identified a cluster of four chicken orthologous CATH genes (CjCATH3, CjCATH2, CjCATHB1, and CjCATH1) located within approximately $13 \mathrm{~kb}$ of one another [10]. This cluster was highly conserved in synteny with chickens. Therefore, investigation of polymorphisms and the function of quail CATHs may contribute to improving the anti-disease properties of chickens and other pheasants.

The identification of amino acid substitutions in mediators of innate immune responses can help to elucidate the relationship between host genomic variations and protection against invading pathogens $[20,21,22,23]$. Single nucleotide polymorphisms (SNPs) and other mutations in HDP genes can affect the function of HDPs and cause differential susceptibility to infections $[23,24,25,26]$.

Multiple CATHs have been identified in a variety of vertebrate species but not in euarchontoglires (e.g., primates, rabbits, and rodents) and carnivores (e.g., cats and dogs) [4]. CATHs are polymorphic in nature, with several known sequence variations in frogs [27], buffalos [28], and humans [29]. Furthermore, copy number variations have been reported in $C A T H$ genes in cattle [30]. However, no relationships between the identified genetic variations and function of CATH peptides have been reported to date. In 2017, we provided the first report of amino acid substitutions in avian CATHs from Japanese quail [10]. Analyses using two different software programs predicted that amino acid substitutions in the Japanese quail variants $\mathrm{CATH} 2$ and $\mathrm{B} 1$ (CjCATH2 and B1) would have potentially significant deleterious effects on peptide function [10]. Although sequence variations have been reported in CATH peptides of other species, the effects of polymorphisms in Japanese quail CATH genes on peptide function have not been reported to date. Thus, the aim of this study was to characterize CATH diversity in Japanese quail and determine the effects of amino acid substitutions on peptide function. We identified the haplotypes and amino acid substitutions in the CATH gene cluster of Japanese quail (CjCATH) and characterized the effects of amino acid substitutions on antimicrobial activity.

\section{Methods}

\section{Animal care}


The management of Japanese quail and all procedures involving animals in the present study were performed in accordance with the Animal Experimental Guidelines of Tokyo University of Agriculture.

\section{Japanese quail}

A total of 99 adult quail representing six strains (18 A [high-immunoglobulin G (lgG)], 15 B [lowIgG], $15 \mathrm{~K}$ [dark], $19 \mathrm{ND}$ [neuron disease], $16 \mathrm{P}$ [panda], and $16 \mathrm{Y}$ [yellow]) were maintained in the Laboratory of Animal Physiology at the Tokyo University of Agriculture, Tokyo, Japan [44,45].

\section{Nucleic acid isolation}

Peripheral blood samples were collected from the jugular vein. Genomic DNA was isolated from peripheral red blood cells using a DNeasy Blood and Tissue kit (QIAGEN) according to the manufacturer's protocol.

\section{Amplification of genomic fragments of four CjCATH genes}

Four fragments (approximately 325 to $517 \mathrm{bp}$ ) were amplified using four primer pairs (Additional file 6) designed based on each CjCATH gene (GenBank accession no. LC136907). Each 50- $\mu \mathrm{L}$ PCR reaction mixture contained $50 \mathrm{ng}$ of quail genomic DNA, $1.25 \mathrm{U}$ of Prime STAR ${ }^{\circledR} \mathrm{GXL}$ DNA polymerase (Takara), $1 \times$ PrimeSTAR ${ }^{\circledR} \mathrm{GXL}$ buffer $\left(\mathrm{Mg}^{2+}\right.$ concentration $\left.1 \mathrm{mmol} / \mathrm{L}\right), 0.2 \mathrm{mmol} / \mathrm{L}$ of each dNTP, and $0.2 \mu \mathrm{mol} / \mathrm{L}$ of each primer. Cycling parameters were as follows: initial denaturation at $98^{\circ} \mathrm{C}$ for $1 \mathrm{~min}$, followed by 35 cycles of denaturation at $98^{\circ} \mathrm{C}$ for $10 \mathrm{~s}$, annealing at $58^{\circ} \mathrm{C}$ for $15 \mathrm{~s}$, and extension at $68^{\circ} \mathrm{C}$ for $30 \mathrm{~s}$. A $2-\mu \mathrm{L}$ aliquot of each PCR reaction was then analyzed by electrophoresis on a 1.0\% agarose gel. PCR products were purified using a QIAquick PCR Purification kit (QIAGEN) and then subjected to direct sequencing using an ABI Capillary System (Macrogen Research).

\section{Amplicon sequencing using MiSeq}

The partial nucleotide sequences of $C j C A T H 2$ and $B 1$ and $C A T H 3$, including insertions or deletions, were determined using MiSeq (Illumina). The forward and reverse primers included the lllumina adapter overhang nucleotide sequences (Additional file 2). Each 50- $\mu$ L PCR reaction mixture contained $50 \mathrm{ng}$ of quail genomic DNA, $25 \mu \mathrm{L}$ of KAPA HIFI HotStart Ready Mix (KAPA), and $1 \mu \mathrm{M}$ forward and reverse primers. PCR products were purified using an AMPURE XP (Beckman Coulter). The index PCR was conducted using a $50-\mu \mathrm{L}$ mixture consisting of $25 \mu \mathrm{L}$ of KAPA HIFI HotStart Ready Mix, $5 \mu \mathrm{L}$ of Nextera 
XT Index Primer 1 (Illumina), $5 \mu \mathrm{L}$ of Nextera XT Index Primer 2 (Illumina), $10 \mu \mathrm{L}$ of sterile $\mathrm{H}_{2} \mathrm{O}$, and $5 \mu \mathrm{L}$ of first-PCR product. PCR products were purified using AMPURE XP (Beckman Coulter). Library quality was assessed on an Agilent 2200 Tapestation (Agilent Technologies). The libraries were sequenced as paired-end, 300-bp reads using a MiSeq (Illumina) platform according to the manufacturer's instructions. All sequence data associated with this project were submitted to the DNA Data Bank of Japan Sequence Reads Archive (DRA) (DRA006654).

\section{Amplicon sequencing analysis}

The overall quality of the MiSeq reads was evaluated using FastQC. The reads were trimmed $1 \mathrm{bp}$ from both the site and low-quality ends (phred score $<30$ ) using the FASTX tool kit (http://hannonlab.cshl.edu/fastx_toolkit/), and unpaired reads were removed. The MiSeq reads were joined using fastq-join [46] with the option $-\mathrm{p} 5-\mathrm{m} \mathrm{40}$, and adapter trimming was performed using cutadapt 1.2.1 [47] with the option --anywhere=CTGTCTCTTATACACATCT -o 9 -e 0.2 -m 250 -M 600. The joined reads were mapped using BWA [48] with default parameters against the phix sequence (GenBank accession no. NC_001422), creating un-mapped reads for further analysis. Reads containing complete primer sequences were recovered from among the un-mapped reads, and primer sequences were trimmed. Trimmed primer reads with an average quality score $>30$ and without unknown nucleotides were retained for further analysis. High-quality reads were converted to a fasta file, and identical sequences were collapsed using the FASTX tool kit. The BLAST database was generated from each CATH using the makeblastdb program included with the BLAST 2.5.0+ package. BLAST searches (blastn algorithm) were used to identify the most-similar CATH sequences. In the analysis of CATH3, identification as a heterozygote or homozygote was based on whether the second highest number of reads was more than one-third of the highest read number.

\section{Comparison of nucleotide sequences}

PCR product sequences were aligned using ClustalW, and alleles were then identified. Genotypes and haplotypes were estimated using PHASE software, ver. 2 [49].

\section{Comparison of nucleotide and amino acid sequences}

Net charges were calculated using Innovagen's Peptide Property Calculator (https://pepcalc.com/). Hydropathy indexes used the value reported by Kyte \& Doolittle (1982) [50]. The impact of all nonsynonymous SNPs on the function of each CATH peptide was assessed using the PROVEAN (http://provean.jcvi.org/index.php) [32] and PolyPhen-2 [33] software packages. A resulting PROVEAN 
score of less than -2.500 indicated a variant was deleterious, and a score of greater than -2.500 indicated a neutral variant [32].

\section{Antimicrobial region peptide synthesis}

GgCATH peptides were designed based on the mature peptide region (Figure 1). CjCATH peptides were designed from six peptides isolated from the mature peptide region of CjCATHs included in this experiment based on amino acid substitutions (Figure 1). All 10 peptides were synthesized by the Funakoshi Corporation, and all were $>95 \%$ pure. Lyophilized peptides were stored in desiccant at $-20^{\circ} \mathrm{C}$ and dissolved in dimethyl sulfoxide and diluted in $10 \mathrm{mmol} / \mathrm{L}$ phosphate buffer $(\mathrm{pH} 7.2)$ before use.

\section{Antimicrobial activity assay}

Escherichia coli (NRIC 1023) was provided by the Nodai Culture Collection Center and used to assess the antimicrobial activity of the 10 synthesized peptides (Figure 1). Following overnight incubation at $37^{\circ} \mathrm{C}, E$. coli cells were subculture for an additional $2 \mathrm{~h}$ at $37^{\circ} \mathrm{C}$ to the mid-logarithmic phase. The cells were then washed once with $10 \mathrm{mmol} / \mathrm{L}$ phosphate buffer $(\mathrm{pH} 7.2)$ and suspended to a concentration of $1 \times 10^{4}$ colony-forming units $/ \mathrm{mL}$ in the same buffer. Ninety microliters of the $E$. coli suspension were placed in 0.2-mL tubes, followed by the addition of $10 \mu \mathrm{L}$ of serially diluted peptide in triplicate. After a 3-h incubation at $37^{\circ} \mathrm{C}$, the cultures were plated onto LENNOX L BROTH agar, incubated overnight at $37^{\circ} \mathrm{C}$, and then the number of surviving bacterial colonies was determined.

\section{Statistical analyses}

Antimicrobial activity was analyzed using a one-way analysis of variance, followed by the HolmBonferroni test for unpaired data. Differences with a $P$ value of $<0.05$ were considered statistically significant.

\section{Results}

\section{Sequence diversity}

To assess allelic diversity in the CjCATH mature peptide-encoding regions, PCR products were amplified from 99 quail specimens representing six strains. A total of 10 SNPs were detected, 5 each at intron3 and exon4 between partial exon3 and partial exon4 of the CjCATH3 locus (325 bp) (Additional file 1). However, any SNPs did not exist in the mature peptide region. Six alleles (CjCATH3*01 *06: GenBank accession nos. LC426739-LC426744) were identified based on these 10 SNPs of CjCATH3 (Table 1). 
These six alleles included three major alleles (CjCATH3*01 [35.9\%], CjCATH3*02 [26.8\%], and CjCATH3*03 [16.2\%]) and three minor alleles (CjCATH1*04 [9.1\%], CjCATH3*05 [8.1\%], and CjCATH3*06 [4.0\%]). CjCATH ${ }^{*} 04$ and $C j C A T H 3{ }^{*} 06$ were specifically detected in strains $\mathrm{K}$ and $\mathrm{B}$, respectively (Table 1 ).

A total of 11 SNPs were detected, 1 at intron3 and 10 at exon4 (mature peptide region) between partial exon3 and partial exon4 of the CjCATH2 locus ( $464 \mathrm{bp}$ ) (Additional file 2). Four alleles (CjCATH2*01 *04: GenBank accession nos. LC426735-LC426738) were identified based on these 11 SNPs of CjCATH2 (Table 1). Ten SNPs in the mature peptide-encoding region were identified, including six that were nonsynonymous. This resulted in the detection of six amino acid substitutions: lle140Val (1140V), lle145Phe (1145F), Ala147Thr (A147T), Gln148His (Q148H), lle149Val (1149V), and Gly150Ala (G150A) (Figure 1). These four alleles included two major alleles (CjCATH2*01 [61.1\%] and CjCATH2*02 [25.8\%[) and two minor alleles (CjCATH2*03 [4.0\%] and CjCATH2*04 [9.1\%]). CjCATH2*03 and CjCATH2*04 were specifically detected in strains $B$ and $\mathrm{K}$, respectively (Table 1 ).

A total of five SNPs were detected, three at intron3 and two at exon4 (mature peptide-encoding region) between partial exon3 and partial exon4 of the CjCATHB1 locus (509 bp) (Additional file 3). Four alleles (CjCATHB1*01 *04: GenBank accession nos. LC426745-LC426748) were identified based on these five SNPs of CjCATHB1 (Table 1).

non-synonymous. These four alleles included three major alleles (CjCATHB1*01 [34.3\%], CjCATHB1*02 [25.8\%], and CjCATHB1*03 [30.8\%]) and one minor allele (CjCATHB1*04 [9.1\%]). CjCATHB1*04 was specifically detected in strain $\mathrm{K}$ (Table 1 ).

A total of seven SNPs were detected, five at intron3 and two at exon4 between partial exon3 and partial exon4 of the CjCATH1 locus (517 bp) (Additional file 4). One SNP with synonymous substitution was detected in the mature peptide-encoding region. Six alleles (CjCATH1*01 *06: GenBank accession nos. LC426729-LC426734) were identified based on these seven SNPs of CjCATH1 (Table 1). These six alleles included three major alleles (CjCATH1*01 [36.4\%], CjCATH1*02 [25.8\%], and CjCATH1*03 [18.7\%]) and three minor alleles (CjCATH1*04 [9.1\%], CjCATH1*05 [6.1\%], and CjCATH1*06 [4.0\%]). CjCATH1*04 and CjCATH $1 * 06$ were specifically detected in strains $\mathrm{K}$ and $\mathrm{B}$, respectively (Table 1 ).

A comparison of the CATH3, CATH2, CATHB1, and CATH1 genes in the 99 quail specimens revealed six, four, four, and six alleles, respectively. We identified nine haplotypes (HT1-HT9) based on combinations of the CATH3, CATH2, CATHB1, and CATH1 alleles (Table 1). HT1 was the primary haplotype in strains ND and $\mathrm{P}$, whereas $\mathrm{HT} 2$ was the primary haplotype in strain B. Haplotypes HT3 and HT4 were detected only in strain ND, and HT9 was found only in strain B. HT7 was a major haplotype specific to strain $\mathrm{K}$.

\section{Properties (Figure 1)}

The amino acid sequence (29 amino acid residues [AA]) of the mature CjCATH3 peptide matched that of GgCATH3. Nine of the $32 \mathrm{AA}$ of the sequence of the mature CjCATH2 peptide differed from the sequence 
of GgCATH2 (K134R, V136I, I/V140R, I144T, A146T, A/T147I, V/I149G, G/A150S, and S151A). The net charge of the C-terminal $15 \mathrm{AA}$ of $\mathrm{CjCATH} 2$ was lower than that of GgCATH2. Conversely, the hydropathy index of CjCATH2 was higher than that of GgCATH2. No significant inter-allelic differences were found in terms of net charge or hydropathy index among the four alleles of CjCATH2. The PROVEAN and PolyPhen-2 software programs were used to evaluate the possibility that six amino acid substitutions among the four alleles of CjCATH2 constituted missense mutations that could adversely affect the function of the peptide. These analyses suggested that the $1145 \mathrm{~F}$ and Q148H substitutions detected in CjCATH2*02 would have an adverse effect on the function of the peptide (Additional file 5).

Of the $40 \mathrm{AA}$ of the mature CjCATHB1 peptide, nine were different from the sequence of GgCATHB1 (T251I, R254W, W257L, D258N, R262K, H271Y, R275H, I278V, and S279T). Although no significant difference in net charge between the $15 \mathrm{~N}$-terminal AA of both CjCATHB1s and GgCATHB1 was detected, the N-terminal hydropathy index of CjCATHB1 was lower than that of GgCATHB1. The net charge of the Cterminal 15 AA of CjCATHB1s was higher than that of the C-terminal 15 amino acid residues GgCATHB1, but there was no significant difference in the C-terminal hydropathy index between CjCATHB1s and GgCATHB1. No significant inter-allelic differences in terms of net charge and hydropathy index of the mature peptides were found among the four alleles of CjCATHB1. However, the PROVEAN and PolyPhen-2 analyses suggested that an amino acid substitution detected in CjCATHB1*04 (Pro245His [P245H]) could affect the function of the CjCATHB1 peptide (Additional file 5).

Among the $26 \mathrm{AA}$ of the sequence of the mature CjCATH1 peptide, two differed from the sequence of GgCATH1 (L128W and R147K). However, no significant differences in net charge or hydropathy index were observed between CjCATH1 and GgCATH1.

\section{Antimicrobial activity (Table 2)}

Synthetic peptides representing all quail and chicken CATHs exhibited concentration-dependent antibacterial activity against $E$. coli. The amino acid sequence of the antimicrobial region of both chicken and Japanese quail CATH3 was the same. However, the amino acid sequences of CATH2, CATHB1 and CATH1 in the quail and chicken differed from each other.

At a concentration of 0.1 to $1 \mu \mathrm{M}$, the antimicrobial activity of CjCATH2s against $E$. coli tended to be higher than that of GgCATH2. The antimicrobial activity of $\mathrm{CjCATH} 2{ }^{*} 01 * 03$ and $\mathrm{CjCATH} 2 * 02$ in particular was significantly more potent than that of GgCATH2. However, there were minimal differences in the antimicrobial activity of $\mathrm{CjCATH} 2$ and GgCATH2 against E. coli at concentrations of 10 and $100 \mu \mathrm{M}$.

At concentrations of 0.1 to $1 \mu \mathrm{M}$, the antimicrobial activity of CjCATHB1s against $E$. coli tended to be higher than that of GgCATHB1. The antimicrobial activity of CjCATHB $1 * 01 * 02 * 03$ at a concentration of $0.5 \mu \mathrm{M}$ was significantly more potent than that of GgCATHB1. However, there were minimal differences in the antimicrobial activity of CjCATHB1 and GgCATHB1 against E. coli at concentrations of 10 and 100 
$\mu \mathrm{M}$. In addition, there were minimal differences in the antimicrobial activity of CjCATH1 and GgCATH1 against $E$. coli at all concentrations examined.

At a peptide concentration of $0.1 \mu \mathrm{M}$, CjCATH3 (=GgCATH3), CjCATH1, and GgCATH1 exhibited significantly more-potent antimicrobial activity against $E$. coli than GgCATH2, CjCATHB1, and GgCATHB1. At a peptide concentration of $0.5 \mu \mathrm{M}, \mathrm{CjCATH} 3, \mathrm{CjCATH} 2 \mathrm{~s}$, CjCATHB $1 * 01 * 02 * 03, \mathrm{CjCATH} 1$, and GgCATH 1 exhibited significantly more-potent antimicrobial activity against $E$. coli than GgCATH2 and GgCATHB1. At a peptide concentration of $1 \mu \mathrm{M}, \mathrm{CjCATH} 2{ }^{*} 01 * 03$ and CjCATH1 exhibited significantly more-potent antimicrobial activity against $E$. coli than GgCATH2 and GgCATHB1. In contrast, at a peptide concentration of $10 \mu \mathrm{M}$, no differences between CATH-derived peptides were observed in terms of antimicrobial activity against $E$. coli. At a CATH-derived peptide concentration of $100 \mu \mathrm{M}$, no survival of $E$. coli was observed.

\section{Discussion}

In nature, CATHs are polymorphic, exhibiting several known sequence variants in frogs [27], buffalos [28], and humans [29]. In addition, copy number variations have been reported in $C A T H$ genes in cattle [30]. No polymorphisms were detected in the amino acid sequence of the mature antimicrobial peptide from Japanese quail liver-expressed antimicrobial peptide 2 (CjLEAP-2), however, and the sequence was found to be the same as that of chicken GgLEAP-2. This sequence similarity suggests that LEAP-2 is highly conserved in birds and may therefore play an as yet unidentified key role in mediating innate immunity [31]. The amino acid sequences of mature CjCATH3 and GgCATH3 peptides are identical, and the similarity between $\mathrm{CjCATH} 1$ and $\mathrm{GgCATH} 1$ is $>90 \%$. Furthermore, CjCATH2/GgCATH2 and CjCATHB1/GgCATHB1 exhibit $83.1 \%$ and $68.7 \%$ similarity, respectively [10]. These data thus suggest that, similar to CjLEAP-2, CATH1 and CATH3 exhibit high sensitivity in terms of recognition of universally present antigens. The amino acid substitutions identified in CATH2 and CATHB1 may be associated with enhanced antigen sensitivity. The observed less-potent antimicrobial activity of CATH2 and CATHB1 against $E$. coli compared with CATH3 and CATH1 supports this hypothesis. Moreover, amino acid substitutions in both CjCATH2 and CjCATHB1 were shown to affect these peptides' antimicrobial activity against $E$. coli. These results were supported by analyses using two different software programs that predicted the amino acid substitutions in CjCATH2 and CjCATHB1 would adversely affect peptide function (PROVEAN:32 and PolyPhen-2区33).

Previous studies focusing on ostrich defensins and avian NK-lysin identified an association between membrane leakage/microbicidal activity and peptide charge [34,35]. However, this association has not been verified with quail and chicken CATH2. CjCATH2 has a lower net charge than GgCATH2 but exhibited more-potent antimicrobial activity at concentrations of $0.1,0.5$, and $1 \mu \mathrm{M}$. Hydrophobicity is thought to be an important mediator of the interaction of the CATHs with bacterial membranes [36,37]. Loss of the first tryptophan residue in GgCATH1 diminishes the peptide's antimicrobial activity, indicating the importance of this hydrophobic residue for the activity of the peptide [18]. Loss of the more-hydrophobic C-terminal residue in GgCATH2 analogues also results in diminished bactericidal activity [38], although C-terminal 
truncation of a GgCATH2 analogue (C1-15 mature peptide) enhanced the antibacterial activity [39]. Interestingly, N-terminal truncation leaving only the hydrophobic $\mathrm{C}$-terminal alpha-helix resulted in almost complete loss of antibacterial activity, probably because the first interaction with bacterial cells involves the polar portions of the $\mathrm{N}$ - and $\mathrm{C}$-termini $[38,39]$. Substitution of phenylalanine with the morehydrophobic tryptophan in the C1-15 mature peptides resulted in enhanced bactericidal activity and better stability in the presence of high salt concentrations [40]. Additionally, substitution of tyrosine with an alanine in a cecropin A-magainin-2 fusion peptide resulted in markedly reduced antibacterial activity $[41,42]$.

In the present study, the antimicrobial activity of the three CjCATH2 peptides against $E$. coli was higher than that of the GgCATH2 peptide, presumably because the $\mathrm{C}$-terminal hydrophobicity of CjCATH2 was higher than that of GgCATH2. However, differences in the C-terminal hydrophobicity among the three CjCATH2 peptides had no apparent effect on the peptides' antimicrobial activity. Substitution of isoleucine with phenylalanine in phenol soluble modulin a (PSMa) greatly reduced antimicrobial activity against Legionella pneumophila [43], whereas the antimicrobial activity of warnericine RK against $L$. pneumophila was not affected by this substitution [43]. These data thus suggest that the antibacterial activity of CATH2 is mediated by some factor other than hydrophobicity.

The antimicrobial activity of CjCATHB1 peptides against E. coli was more potent than that of GgCATHB1 peptides. The hydrophobicity of the mature CjCATHB1 peptides was lower than that of GgCATHB1. Conversely, the net charge of the CjCATHB $1{ }^{*} 01{ }^{*} 02 * 03$ and CjCATHBB $1 * 04$ peptides was higher than that of the GgCATHB1 peptide. These observations suggest that the antibacterial activity of CATHB1 is affected by net charge. In contrast, the antimicrobial activity of CjCATHB1 peptides differed at a concentration of $10 \mathrm{nM}$. Substitution of proline with a more-hydrophilic histidine as the first amino acid of mature CjCATHB1 enhanced the peptide's antimicrobial activity (Table 2). These results are in agreement with those of previous reports indicating that the antimicrobial activity of avian CATHs is affected by hydrophobicity $[18,38,39,40]$. Therefore, the antimicrobial activity of CjCATHB1 may be affected by hydrophobicity in a manner similar to CjCATH1 and CjCATH2.

\section{Conclusions}

In this study, several SNPs in the mature peptide-encoding regions of CjCATH genes were identified. CjCATH2 contains six non-synonymous SNPs, whereas CjCATHB1 contains one; three and two different peptides, respectively, were synthesized based on each allele. The antimicrobial activity of the three CjCATH2 peptides against E. coli determined in vitro was more potent than that of GgCATH2 peptide, suggesting that hydrophobicity is important to antimicrobial activity. The antimicrobial activity of the two CjCATHB1 peptides was more potent than that of GgCATHB1 peptide, suggesting that net charge is important to antimicrobial activity. The difference in antimicrobial activity of the two CjCATHB1 peptides was associated with substitution of proline with a more-hydrophilic histidine residue as the first amino acid. The amino acid substitutions identified in our study affect the antimicrobial activity of CATHs, which could in turn affect the binding affinity for LPS and ultimately the effectiveness of innate immune 
responses in this commercially important species. Overall, these results suggest that genetic diversity may affect resistance to infection by pathogens in Japanese quail.

\section{Abbreviations}

Cj: Coturnix japonica; CATH: cathelicidin; CATH1: cathelicidin 1;

CATH2: cathelicidin 2; CATH3: cathelicidin 3; CATHB1: cathelicidin B1; SNP: single nucleotide polymorphism; Gg: Gallus gallus;

LPS: lipopolysaccharide

\section{References}

1. Zasloff M.: Antimicrobial peptides of multicellular organisms. Nature 2002;415:389-395.

2. Yang D, Biragyn A, Hoover DM, Lubkowski J, Oppenheim JJ.: Multiple roles of antimicrobial defensins, cathelicidins, and eosinophil-derived neurotoxin in host defense. Annu Rev Immunol. 2004;22:181-215.

3. Zanetti M.: Cathelicidins, multifunctional peptides of the innate immunity. J Leukoc Biol. 2004;75:3948.

4. Zhang G, Sunkara LT.: Avian antimicrobial host defense peptides: from biology to therapeutic applications. Pharmaceuticals (Basel). 2014;7:220-247.

5. Zanetti M.: The role of cathelicidins in the innate host defenses of mammals. Curr Issues Mol Biol. 2005;7:179-96.

6. Roby KD, Di Nardo A.: Innate immunity and the role of the antimicrobial peptide cathelicidin in inflammatory skin disease. Drug Discov Today Dis Mech. 2013;10:e79-e82.

7. Ageitos, JM, Sánchez-Pérez A, Calo-Mata P, Villa TG.: Antimicrobial peptides (AMPs): Ancient compounds that represent novel weapons in the fight against bacteria. Biochem Pharmacol. 2017;133:117-138.

8. Coorens M, Banaschewski BJH, Baer BJ, Yamashita C, van Dijk A, Haagsman HP, Veldhuizen RAW, Veldhuizen EJA. Killing of P.: aeruginosa by chicken cathelicidin-2 is immunogenically silent, preventing lung inflammation in vivo. Infect Immun. 2017;85:e00546-17.

9. Cheng Y, Prickett MD, Gutowska W, Kuo R, Belov K, Burt DW.: Evolution of the avian $\beta$-defensin and cathelicidin genes. BMC Evol Biol. 2015;15:188.

10. Ishige $\mathrm{T}$, Hara H, Hirano $\mathrm{T}$, Kono $\mathrm{T}$, Hanzawa K.: Characterization of the cathelicidin cluster in the Japanese quail (Coturnix japonica). Anim Sci J. 2017;88:1249-1257.

11. Xiao Y, Cai Y, Bommineni YR, Fernando SC, Prakash O, Gilliland SE, Zhang G.: Identification and functional characterization of three chicken cathelicidins with potent antimicrobial activity. J Biol Chem. 2006;281:2858-2867. 
12. Lynn DJ, Higgs R, Gaines S, Tierney J, James T, Lloyd AT, Fares MA, Mulcahy G, O'Farrelly C.: Bioinformatic discovery and initial characterisation of nine novel antimicrobial peptide genes in the chicken. Immunogenetics. 2004;56:170-177.

13. van Dijk A, Veldhuizen EJ, van Asten AJ, Haagsman HP.: CMAP27, a novel chicken cathelicidin-like antimicrobial protein. Vet Immunol Immunopathol. 2005;106:321-327.

14. Achanta M, Sunkara LT, Dai G, Bommineni YR, Jiang W, Zhang G.: Tissue expression and developmental regulation of chicken cathelicidin antimicrobial peptides. J Anim Sci Biotechnol. 2012;3:15.

15. Goitsuka R, Chen CL, Benyon L, Asano Y, Kitamura D, Cooper MD.: Chicken cathelicidin-B1, an antimicrobial guardian at the mucosal M cell gateway. Proc Natl Acad Sci USA. 2007;104:1506315068.

16. Meade KG, Higgs R, Lloyd AT, Giles S, O'Farrelly C.: Differential antimicrobial peptide gene expression patterns during early chicken embryological development. Dev Comp Immunol. 2009;33:516-524.

17. Xiao Y, Dai H, Bommineni YR, Soulages JL, Gong YX, Prakash O, Zhang G.: Structure-activity relationships of fowlicidin-1, a cathelicidin antimicrobial peptide in chicken. FEBS J. 2006;273:25812593.

18. Bommineni YR, Dai H, Gong YX, Soulages JL, Fernando SC, Desilva U, Prakash O, Zhang G.: Fowlicidin-3 is an a-helical cationic host defense peptide with potent antibacterial and lipopolysaccharide-neutralizing activities. FEBS J. 2007;274:418-428.

19. van Dijk A, Tersteeg-Zijderveld MH, Tjeerdsma-van Bokhoven JL, Jansman AJ, Veldhuizen EJ, Haagsman HP.: Chicken heterophils are recruited to the site of Salmonella infection and release antibacterial mature Cathelicidin-2 upon stimulation with LPS. Mol Immunol. 2009;46:1517-1526.

20. Barreiro LB, Tailleux L, Pai AA, Gicquel B, Marioni JC, Gilad Y.: Deciphering the genetic architecture of variation in the immune response to Mycobacterium tuberculosis infection. Proc Natl Acad Sci USA. 2012;109:1204-1209.

21. Seabury CM, Seabury PM, Decker JE, Schnabel RD, Taylor JF, Womack JE.: Diversity and evolution of 11 innate immune genes in Bos taurus taurus and Bos taurus indicus cattle. Proc Natl Acad Sci USA. 2010;107:151-156.

22. Pankratz VS, Vierkant RA, O'Byrne MM, Ovsyannikova IG, Poland GA.: Associations between SNPs in candidate immune-relevant genes and rubella antibody levels: A multigenic assessment. BMC Immunol. 2010;11:48.

23. Hasenstein JR, Lamont SJ. Chicken gallinacin gene cluster associated with Salmonella response in advanced intercross line. Avian Dis. 2007;51:561-567.

24. Rivas-Santiago B, Serrano CJ, Enciso-Moreno JA.: Susceptibility to infectious diseases based on antimicrobial peptide production. Infect Immun. 2009;77:4690-4695.

25. Matsushita I, Hasegawa K, Nakata K, Yasuda K, Tokunaga K, Keicho N.: Genetic variants of human beta-defensin-1 and chronic obstructive pulmonary disease. Biochem Biophys Res Commun. 2002;291:17-22. 
26. Hellgren O, Sheldon BC, Buckling A.: In vitro tests of natural allelic variation of innate immune genes (avian $\beta$-defensins) reveal functional differences in microbial inhibition. J Evol Biol. 2010;23:27262730.

27. Yu H, Cai S, Gao J, Zhang S, Lu Y, Qiao X, Yang H, Wang Y.: Identification and polymorphism discovery of the cathelicidins, Lf-CATHs in ranid amphibian (Limnonectes fragilis). FEBS J. 2013 ;280:6022-6032.

28. Brahma B, Patra MC, Karri S, Chopra M, Mishra P, De BC, Kumar S, Mahanty S, Thakur K, Poluri KM, Datta TK, De S.: Diversity, antimicrobial action and structure-activity relationship of buffalo Cathelicidins. PLoS One. 2015;19:e0144741.

29. López Campos GN, Velarde Félix JS, Sandoval Ramírez L, Cazares Salazar S, Corona Nakamura AL, Amaya Tapia G, Prado Montes de Oca E.: Polymorphism in cathelicidin gene (CAMP) that alters Hypoxia-inducible factor (HIF-1a:: ARNT) binding is not associated with tuberculosis. Int $\mathrm{J}$ Immunogenet. 2014;41:54-62.

30. Chen L, Chamberlain AJ, Reich CM, Daetwyler HD, Hayes BJ.: Detection and validation of structural variations in bovine whole-genome sequence data. Genet Sel Evol. 2017;49:13.

31. Ishige $\mathrm{T}$, Hara H, Hirano $\mathrm{T}$, Kono $\mathrm{T}$, Hanzawa K.: Characterization and expression of non-polymorphic liver expressed antimicrobial peptide 2: LEAP-2 in the Japanese quail, Coturnix japonica. Anim Sci J. 2016;87:1182-1187.

32. Choi Y, Sims GE, Murphy S, Miller JR, Chan AP.: Predicting the functional effect of amino acid substitutions and indels. PLoS One. 2012;7:e46688.

33. Adzhubei IA, Schmidt S, Peshkin L, Ramensky VE, Gerasimova A, Bork P, Kondrashov AS, Sunyaev SR.: A method and server for predicting damaging missense mutations. Nat Methods. 2010;7:248249.

34. Lee MO, Kim EH, Jang HJ, Park MN, Woo HJ, Han JY, Womack JE.: Effects of a single nucleotide polymorphism in the chicken NK-lysin gene on antimicrobial activity and cytotoxicity of cancer cells. Proc Natl Acad Sci USA. 2012;109:12087-12092.

35. Sugiarto H, Yu PL.: Identification of three novel ostricacins: an update on the phylogenetic perspective of $\beta$-defensins. Int J Antimicrob Agents. 2006;27:229-235.

36. Nicolas P.: Multifunctional host defense peptides: intracellular-targeting antimicrobial peptides. FEBS J. 2009;276:6483-6496.

37. Oren Z, Shai Y.: Mode of action of linear amphipathic a-helical antimicrobial peptides. Biopolymers. 1998;47:451-463.

38. Xiao Y, Dai H, Bommineni YR, Soulages JL, Gong YX, Prakash O, Zhang G.: The central kink region of fowlicidin-2, an a-helical host defense peptide, is critically involved in bacterial killing and endotoxin neutralization. J Innate Immun. 2009;1:268-280.

39. van Dijk A, Molhoek EM, Veldhuizen EJ, Bokhoven JL, Wagendorp E, Bikker F, Haagsman HP.: Identification of chicken cathelicidin-2 core elements involved in antibacterial and immunomodulatory activities. Mol Immunol. 2009;46:2465-2473. 
40. Molhoek EM, van Dijk A, Veldhuizen EJ, Haagsman HP, Bikker FJ.: A cathelicidin-2-derived peptide effectively impairs Staphylococcus epidermidis biofilms. Int J Antimicrob Agents. 2010;37:476-479.

41. Nan YH, Bang JK, Jacob B, Park IS, Shin SY.: Prokaryotic selectivity, anti-endotoxic activity and protease stability of diastereomeric and enantiomeric analogs of human antimicrobial peptide LL-37. Bulletin of the Korean Chemical Society. 2012;33:2883-2889.

42. Dathe $M$, Nikolenko H, Meyer J, Beyermann M, Bienert M.: Optimization of the antimicrobial activity of magainin peptides by modification of charge. FEBS letters. 2001;501:146-150.

43. Marchand A, Augenstreich J, Loiseau C, Verdon J, Lecomte S, Berjeaud JM.: Effect of amino acid substitution in the staphylococcal peptides warnericin RK and PSMa on their anti-Legionella and hemolytic activities. Mol Cell Biochem. 2015;405:159-167.

44. Shiina T, Ando A, Imanishi T, Kawata H, Hanzawa K, Gojobori T, Inoko H, Watanabe S.: Isolation and characterization of CDNA clones for Japanese quail (Coturnix japonica) major histocompatibility complex (MhcCoja) class I molecules. Immunogenetics. 1995;42:213-216.

45. Suzuki S, Hosomichi K, Yokoyama K, Tsuda K, Hara H, Yoshida Y, Fujiwara A, Mizutani M, Shiina T, Kono T, Hanzawa K.: Primary analysis of DNA polymorphisms in the TRIM region (MHC subregion) of the $\mathrm{J}$ apanese quail, C oturnix japonica. Anim Sci J. 2013;84:90-96.

46. Esposito A, Ahmed E, Ciccazzo S, Sikorski J, Overmann J, Holmström SJ, Brusetti L.: Comparison of rock varnish bacterial communities with surrounding non-varnished rock surfaces: taxon-specific analysis and morphological description. Microb Ecol. 2015;70:741-750.

47. Martin M.: Cutadapt removes adapter sequences from high-throughput sequencing reads. EMBnet J. 2011;17:10-12.

48. Li H, Durbin R.: Fast and accurate short read alignment with Burrows-Wheeler Transform. Bioinformatics. 2009;25:1754-1760.

49. Stephens M, Scheet P.: Accounting for decay of linkage disequilibrium in haplotype inference and missing-data imputation. Am J Hum Genet. 2005;76:449-462.

50. Kyte J, Doolittle RF.: A simple method for displaying the hydropathic character of a protein. J Mol Biol. 1982;157:105-132.

\section{Declarations}

\section{Ethics approval}

The management of Japanese quail and all experimental procedures undertaken in the present study were performed according to the recommendations of the Guidelines by the ethics committee for animal experiments of the Tokyo University of Agriculture (Tokyo, Japan). All Japanese quails were maintained in order to preserve the strain after the study. 
Not applicable.

\section{Availability of data and material}

The data sets supporting the results of this article are included within the article and its additional files. All Next Generation Sequencer of sequences data associated with this project were submitted to the DNA Data Bank of Japan Sequence Reads Archive (DRA) (DRA006654).

\section{Competing interests}

The authors declare that they have no competing interests.

\section{Funding}

This research was supported in part by the Advanced Research Project Type A, Tokyo University of Agriculture (No.02, 2006-2008), and MEXT-Supported Program for the Strategic Research Foundation at Private Universities, 2013-2017 (S1311017).

\section{Author information}

\section{Affiliations}

NODAI Genome Research Center, Tokyo University of Agriculture, Sakuragaoka, Setagaya, Tokyo, 1568502, Japan

Taichiro Ishige

Department of Animal Science, Tokyo University of Agriculture, Funako, Atsugi, Kanagawa, 243-0034, Japan

Hiromi Hara, Hirano Takashi, Hanzawa Kei

Department of Bioscience, Tokyo University of Agriculture, Tokyo, Sakuragaoka, Setagaya, Tokyo, 1568502, Japan

Tomohiro Kono 


\section{Authors' contributions}

$\mathrm{TI}$ and $\mathrm{KH}$ conceived and designed the study. TI collected data, performed all experiments, and wrote the manuscript. $\mathrm{HH}, \mathrm{HT}$ and TK assisted in drafting the manuscript. All authors read and approved the final manuscript.

\section{ACKNOWLEDGMENTS}

Not Applicable.

\section{Tables}


Table 1. CjCATH haplotype frequencies in six Japanese quail strains.

\begin{tabular}{|c|c|c|c|c|c|c|c|c|}
\hline \multirow[t]{2}{*}{ Haplotype } & \multirow{2}{*}{$\begin{array}{l}\text { Locus } \\
\text { CATH3-2-B1-1 }\end{array}$} & \multicolumn{6}{|c|}{ Strain (number) } & \multirow[t]{2}{*}{ Total } \\
\hline & & $\mathrm{A}$ & B & $\mathrm{K}$ & ND & $\mathrm{P}$ & $\mathrm{Y}$ & \\
\hline & & (18) & (15) & (15) & (19) & (16) & (16) & (99) \\
\hline HT1 & *01-*01-*01-*01 & 0.36 & - & 0.07 & 0.84 & 0.56 & 0.19 & 0.36 \\
\hline HT2 & *02-*02-*02-*02 & 0.19 & 0.73 & 0.10 & - & 0.44 & 0.16 & 0.26 \\
\hline HT3 & *02-*01-*01-*01 & - & - & - & 0.03 & - & - & 0.01 \\
\hline HT4 & *02-*01-*03-*03 & - & - & - & 0.03 & - & - & 0.01 \\
\hline HT5 & *03-*01-*03-*03 & 0.39 & - & - & - & - & 0.19 & 0.10 \\
\hline HT6 & *03-*01-*03-*05 & 0.06 & - & 0.23 & 0.03 & - & 0.06 & 0.06 \\
\hline HT7 & $* 04-* 04-* 04-* 04$ & - & - & \#\#\# & - & - & - & 0.09 \\
\hline HT8 & *05-*01-*03-*03 & - & - & - & 0.08 & - & 0.41 & 0.08 \\
\hline HT9 & *06-*03-*03-*06 & - & 0.27 & - & - & - & - & 0.04 \\
\hline
\end{tabular}

Red: majority frequency (>50\%) of strain, blue box: strain specific, -: Not observed 
Table 2. Effect of CATH-derived synthetic peptides on viability of the gram-negative bacterium Escherichia coli.

peptide concentration $(\mu \mathrm{M})$

$\begin{array}{llllll}\text { Locus } & \text { Peptide (See Figure } & 0.1 & 0.5 & 1 & 10\end{array}$

CATH3
CjCATH3 (GgCATH3)
$42.9 \pm 1.7^{\text {ay }}$
$22.4 \pm 7.4^{\text {by }}$
$13.1 \pm 0.5^{\text {bcxyz }}$
$6.3 \pm 0.9^{\mathrm{cw}} \quad 0$

CATH2

\begin{tabular}{lccccc} 
CjCATH2*01*03 & $66.9 \pm 2.7^{\text {bcwxy }}$ & $17.4 \pm 5.6^{\text {dey }}$ & $6.4 \pm 1.5^{\text {ez }}$ & $4.7 \pm 0.7^{\text {ew }}$ & 0 \\
\hline CjCATH2*02 & $76.7 \pm 4.3^{\text {bcw }}{ }^{*}$ & $18.7 \pm 3.5^{\text {dey }}$ & $6.1 \pm 0.7^{\mathrm{ez}}$ & $4.8 \pm 1.0^{\text {ew }}$ & 0 \\
\hline CjCATH2*04 & $79.3 \pm 6.6^{\text {abwxy }}$ & $24.6 \pm 4.8^{\text {dey }}$ & $10.5 \pm 2.0^{\text {dexyz }}$ & $6.2 \pm 1.8^{\text {ew }}$ & 0 \\
\hline GgCATH2 & $97.8 \pm 5.4^{\text {aw }}$ & $82.9 \pm 1.8^{\text {abw }}$ & $34.7 \pm 7.1^{\text {cdwx }}$ & $11.0 \pm 4.4^{\text {dew }}$ & 0
\end{tabular}

CATHB1

\begin{tabular}{lccccc} 
CjCATHB $1 * 01 * 02 * 03$ & $92.7 \pm 6.9^{\mathrm{abwx}}$ & $43.0 \pm 0.7^{\text {dew } x y}$ & $31.4 \pm 3.0^{\text {dexy }}$ & $14.2 \pm 2.2^{\text {ew }}$ & 0 \\
\hline CjCATHB $1 * 04$ & $94.4 \pm 0.5^{\mathrm{abwx}}$ & $69.9 \pm 10.9^{\mathrm{abcdwx}}$ & $31.6 \pm 4.3^{\mathrm{dexy}}$ & $12.3 \pm 3.1^{\mathrm{ew}}$ & 0 \\
\hline GgCATHB1 & $97.5 \pm 4.8^{\mathrm{aw}}$ & $83.6 \pm 3.1^{\mathrm{abcw}}$ & $62.6 \pm 9.1^{\mathrm{bcdw}}$ & $8.2 \pm 1.1^{\mathrm{ew}}$ & 0
\end{tabular}

\section{CATH1}

\begin{tabular}{lccccc} 
CjCATH1 & $48.0 \pm 7.8^{\mathrm{ay}}$ & $32.8 \pm 7.8^{\mathrm{abxy}}$ & $8.6 \pm 1.5^{\mathrm{bcyz}}$ & $2.9 \pm 1.1^{\mathrm{cw}}$ & 0 \\
\hline GgCATH1 & $54.4 \pm 9.4^{\mathrm{axy}}$ & $27.4 \pm 9.9^{\mathrm{abcxy}}$ & $13.8 \pm 2.1^{\mathrm{bcxyz}}$ & $5.4 \pm 1.6^{\mathrm{bcw}}$ & 0
\end{tabular}

Results are presented as means \pm SEM $(n=3)$. Means followed by the same small letter in the same row are not significantly different $(\mathrm{P}<0.05)$. Concentration-dependent differences in each CATH: $\mathrm{a}>\mathrm{b}>\mathrm{c}>\mathrm{d}>\mathrm{e}$. Differences between CATHs at each concentration: $\mathrm{w}>\mathrm{x}>\mathrm{y}>\mathrm{z}$. 


\section{Figures}

\begin{tabular}{|c|c|c|c|c|c|c|c|c|c|c|c|}
\hline \multirow{2}{*}{ Peptide } & \multirow{2}{*}{ Allele } & & \multirow{2}{*}{ Sequence } & \multirow{2}{*}{ Length } & \multirow{2}{*}{ MW } & \multicolumn{3}{|c|}{ Net charge } & \multicolumn{3}{|c|}{ Hydropathy index } \\
\hline & & & & & & Full & $\begin{array}{l}\text { N-end } \\
15 A A\end{array}$ & $\begin{array}{l}\text { C-end } \\
15 A A\end{array}$ & Full & $\begin{array}{l}\text { N-end } \\
15 A A\end{array}$ & $\begin{array}{l}\text { C-end } \\
15 A A\end{array}$ \\
\hline CjCATH3 (GgCATH3) $\square$ & $* 01 \sim * 06$ & & $R V K R--F W P L V P V A I N T V A A G I N L Y K A I R R K$ & 29 & 3351.05 & 7.0 & 3.0 & 5.0 & 0.16 & 0.29 & -0.02 \\
\hline $\begin{array}{l}\text { CjCATH } 2 * 01 * 03 \\
\text { CjCATH2 } 202 \square \\
\text { CjCATH2 } 2 * 04 \square \\
\text { GgCATH2 }\end{array}$ & $\begin{array}{l}* 01 \& * 03 \\
* 02 \\
* 04\end{array}$ & & 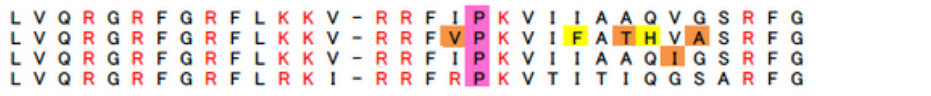 & $\begin{array}{l}32 \\
32 \\
32 \\
32\end{array}$ & $\begin{array}{l}3701.47 \\
3774.52 \\
3715.49 \\
3818.53\end{array}$ & $\begin{array}{r}9.0 \\
9.1 \\
9.0 \\
10.0\end{array}$ & $\begin{array}{l}6.0 \\
6.0 \\
6.0 \\
6.0\end{array}$ & $\begin{array}{l}2.0 \\
2.1 \\
2.0 \\
3.0\end{array}$ & $\begin{array}{r}0.09 \\
0.03 \\
0.10 \\
-0.43\end{array}$ & $\begin{array}{l}-0.57 \\
-0.57 \\
-0.57 \\
-0.59\end{array}$ & $\begin{array}{r}0.88 \\
0.75 \\
0.90 \\
-0.21\end{array}$ \\
\hline $\begin{array}{l}\text { CjCATHB } 1 * 01 * 02 * 03 \square \\
\text { CjCATHB } 1 * 04 \square \\
\text { GgCATHB1D }\end{array}$ & $* 01, * 02 \& * 03$ & $\begin{array}{ll}P & 1 \\
H & 1 \\
P & 1\end{array}$ & 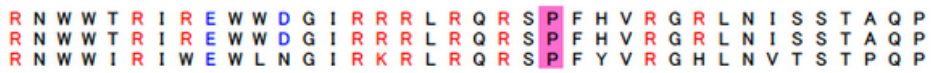 & $\begin{array}{l}40 \\
40 \\
40\end{array}$ & $\begin{array}{l}5055.73 \\
5095.76 \\
5028.79\end{array}$ & $\begin{array}{l}8.1 \\
8.2 \\
7.1\end{array}$ & $\begin{array}{l}1.0 \\
1.1 \\
1.0\end{array}$ & $\begin{array}{l}2.1 \\
2.1 \\
1.1\end{array}$ & $\begin{array}{l}-1.35 \\
-1.40 \\
-0.81\end{array}$ & $\begin{array}{l}-1.42 \\
-1.53 \\
-0.52\end{array}$ & $\begin{array}{l}-0.43 \\
-0.43 \\
-0.45\end{array}$ \\
\hline $\begin{array}{l}\text { CјCATH1D } \\
\text { GgCATH1D }\end{array}$ & $* 01^{\sim} * 06$ & & 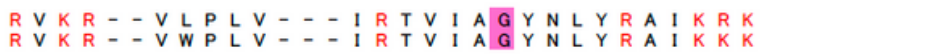 & $\begin{array}{l}26 \\
26\end{array}$ & $\begin{array}{l}3096.81 \\
3141.85\end{array}$ & $\begin{array}{l}8.0 \\
8.0\end{array}$ & $\begin{array}{l}4.0 \\
4.0\end{array}$ & $\begin{array}{l}4.0 \\
4.0\end{array}$ & $\begin{array}{r}0.09 \\
-0.07\end{array}$ & $\begin{array}{l}1.03 \\
0.72\end{array}$ & $\begin{array}{l}-0.23 \\
-0.19\end{array}$ \\
\hline
\end{tabular}

Amino acid sequences of mature peptides were aligned by MEGA6.

Yellow highlight indicates amino acid substitution that were predicted to have possibly damaging effects on protein function.

Orange highlight indicates amino acid substitution that were predicted to have no effects on protein function.

Red and blue letters indicate cationic amino acids (K\& \&) and acidic amino acids (D \& E), respectively.

Length: number of amino acid residues. MW: molecular weight.

Net charge at pH7 calculated by PepCalc.com-Peptide property (https://pepcalc.com/ calculator).

Hydropathy index (Kyte \& Doolittle 1982).

\section{Figure 1}

Sequence diversity and properties of mature quail and chicken CATH peptides.

\section{Supplementary Files}

This is a list of supplementary files associated with this preprint. Click to download.

- Additionalfile1Cath3AlignV6.xlsx

- Additionalfile5AminoAcidSubstitutionV6.xlsx

- Additionalfile2Cath2AlignV6.xlsx

- Additionalfile3CathB1AlignV6.xlsx

- Additionalfile6PrimerPairV7.xlsx

- Additionalfile4Cath1AlignV7.xlsx

- ManuscriptishigeCATH2ndv8resub.docx 